\title{
Research on the Numerical Method and Parametric Study of Constitutive Model of Modified Double Base Propellant
}

\author{
Hongli Wang ${ }^{\text {a)}}$, Jinsheng $\mathrm{Xu}{ }^{\text {b) }}$
}

\author{
School of Mechanical Engineering, Nanjing University of Science and technology, \\ Nanjing 210094, China \\ a)whlyear@163.com; b)xujinsheng@njust.edu.cn
}

Keywords: Constitutive Model, Numerical Method, Parametric Study

\begin{abstract}
In order to characterize uniaxial mechanical properties of modified double base propellant, a one dimensional form of viscoelastic-viscoplastic constitutive model is derived. In order to solve the first-order ordinary differential equations (ODEs) consisted by viscoplastic flow rule and internal state variables evolution, the backward difference formulas (BDF) is applied and achieved by MATLAB procedure.The effect of viscoplastic parameters on viscoplastic creep strain is analyzed by adjusting viscoplastic parameters based on a set of the given parameters, the results show that the greater values of viscoplastic potential energy parameter $\Gamma$ correspond to more flow while $N$ correspond to more flow,the greater values of the hardening function parameters result in less flow.
\end{abstract}

\section{Introduction}

Solid rocket motors is widely used in military weapon and aerospace fields. The structural integrity of the solid propellant grain affect the safety and reliability of the rocket weapon, and is currently the critical task to solid rocket motor. In order to obtain the mechanical properties of solid rocket propellant, many scholars studied the constitutive model of the propellant. XU[1] establishes the thermal-damage-viscoelastic constitutive model to describe the mechanical properties of HTPB composite propellant. DENG[2] studies the temperature dependent viscoelastic mechanics of solid propellant by shear relaxation experiments. Modified double-base propellant, with its very complex mechanical properties is a kind of commonly used solid propellant. SUN [3]deeply analyses quasistatic and dynamic compression mechanics of modified double-base propellant, experimental results show that the yield stress, the initial modulus and the maximum strain are significantly affected by strain rate and temperature. Meng Gonglei[4] proposes a nonlinear viscoelastic constitutive model which includes cumulative damage for nonlinear mechanical behavior of modified double-base propellant.

Modified double-base propellant is a kind of granular material, experimental results show that it has viscoelastic and viscoplastic mechanics. Many scholars studied the viscoelastic and viscoplastic constitutive model of solid material, in the establishment of DARABI[5,6] establish a thermalviscoelastic-viscoplastic constitutive model to describe the mechanical properties of heat asphalt mixtures. KIM[7] compare the Schapery-Perzyna model and Schapery-Valanis modelto describe the viscoelastic and viscoplastic mechanics of particles reinforced material. ZHU[8] and ZHU[9] use backward difference formulas to solve viscoelastic - viscoplastic model in order to obtain optimum model parameters. 
In this study, one dimensional form of viscoelastic-viscoplastic constitutive model of modified double base propellant is derived, and backward difference formulas is used to solve viscoplastic model. Parametric study of constitutive model focued on the creep response of modified double base propellant is analyzed.

\section{Viscoelastic-Viscoplastic Constitutive Model}

In researches on constitutive model of solid materials, the total deformation subjected to an applied stress can be decomposed into recoverable and irrecoverable components. Elastic and viscoelastic models are used to describe recoverable deformation, while plastic and viscoplastic models are employed to model irrecoverable deformation.

Experimental research shows that the deformation of modified double base propellant include recoverable and irrecoverable deformation, where the extent of each is mainly affected by time, temperature, and loading rate. In this study, the total strain of modified double base propellant is decoupled into recoverable viscoelatic strain and irrecoverable viscoplastic strain:

$$
\varepsilon_{i j}=\varepsilon_{i j}^{\mathrm{ve}}+\varepsilon_{i j}^{\mathrm{vp}}
$$

where $\varepsilon_{i j}$ is the total strain tensor; $\varepsilon_{i j}^{\mathrm{ve}}$ is the viscoelastic strain tensor; and $\varepsilon_{i j}^{\mathrm{vp}}$ is the viscoplastic strain tensor.

\section{Viscoelastic Constitutive Model}

In this study, the Schapery's linear viscoelasticity theory is used to describe the viscoelastic response of modified double base propellant, when the loading stress is $\sigma^{\tau}$ :

$$
\varepsilon_{i j}^{\mathrm{ve}, t}=D_{0} \sigma_{i j}^{t}+\int_{0}^{t} \Delta D^{\left(\psi^{t}-\psi^{\tau}\right)} \frac{d\left(\sigma_{i j}^{\tau}\right)}{d \tau} d \tau
$$

where $D_{0}$ is the instantaneous compliance; $\Delta D$ is the transient compliance; $\psi^{t}=t$ when temperature effect isn't considered. The Prony series is used to represent the transient compliance $\Delta D$ for numerical convenience, such that:

$$
\Delta D^{t}=\sum_{n=1}^{\mathrm{N}} D_{n}\left[1-\exp \left(-\lambda_{n} t\right)\right]
$$

\section{Viscoplastic Constitutive Model}

Differentiating Eq. (1) with respect to the time implies:

$$
\dot{\varepsilon}_{i j}=\dot{\varepsilon}_{i j}^{\mathrm{ve}}+\dot{\varepsilon}_{i j}^{\mathrm{vp}}
$$

where the viscoplastic strain rate is expressed as follows:

$$
\dot{\varepsilon}_{i j}^{\mathrm{vp}}=\Gamma\langle\phi(f)\rangle^{N} \frac{\partial g}{\partial \sigma_{i j}}
$$

where $\langle\bullet\rangle$ is McCauley bracket, $\Gamma$ is the viscoplastic viscosity parameter, $\phi$ is the overstress function, $N$ is the viscoplastic rate sensitivity exponent, $g$ is the viscoplastic potential function, overstress function is expressed as follows:

$$
\phi(f)=\left(f / \sigma_{y}^{0}\right)^{N}
$$

Where $\sigma_{y}^{0}$ is reference stress. The Drucker-prager yield surfaces is used: 


$$
f=\tau-\alpha I_{1}-k\left(\varepsilon_{\mathrm{e}}^{\mathrm{vp}}\right)
$$

where $\tau=\sqrt{3 S_{i j} S_{i j} / 2}, I_{1}=\sigma_{k k}, \alpha$ is a material parameter, $k$ is the isotropic hardening function, LEMAITRE and CHABOCHE [10] proposed a isotropic hardening model as follows:

$$
k\left(\varepsilon_{\mathrm{e}}^{\mathrm{vp}}\right)=k_{0}+k_{1}\left(1-\exp \left(-k_{2} \varepsilon_{\mathrm{e}}^{\mathrm{vp}}\right)\right)
$$

where $k_{0}, k_{1}$ and $k_{2}$ are material parameters, $\varepsilon_{\mathrm{e}}^{\mathrm{vp}}$ is effective viscoplastic strain. The effective viscoplastic strain rate $\dot{\varepsilon}_{e}^{v p}$ is expressed as follows[11]:

$$
\dot{\varepsilon}_{e}^{v p}=\left(1+2\left(\frac{0.5+\beta / 3}{1-\beta / 3}\right)\right)^{-1 / 2} \sqrt{\dot{\varepsilon}_{i j}^{v p} \dot{\varepsilon}_{i j}^{v p}}
$$

It can be shown that for the uniaxial state of stresses, $\dot{\varepsilon}_{\mathrm{e}}^{\mathrm{vp}}=\dot{\varepsilon}_{11}^{\mathrm{vp}}$.

The non-associated flow rules are commonly used to describe the viscoplasticity in the studies of material viscoplastic mechanics[11,12,13], in this study the non-associated flow rule is expressed as follows:

$$
g=\tau-\beta I_{1}
$$

where $\beta$ is material parameter, then one can write:

$$
\frac{\partial g}{\partial \sigma_{i j}}=\frac{\partial \tau}{\partial \sigma_{i j}}-\frac{1}{3} \beta \delta_{i j}
$$

\section{Numerical Method of Constitutive Model}

In this paper, the strain-style constitutive model is adopted, the uniaxial creep conditions are studied here for numerical convenience. Under the condition of uniaxial compression, the viscoelastic-viscoplastic model can be transformed to one dimensional form as follows:

Fundamental equation

$$
\dot{\varepsilon}_{\text {total }}=\dot{\varepsilon}_{\mathrm{ve}}+\dot{\varepsilon}_{\mathrm{vp}}
$$

Rules of flow

$$
\left\{\begin{array}{l}
\varepsilon_{\mathrm{ve}}=\sigma\left\{D_{0}+\sum_{n=1}^{\mathrm{N}} D_{n}\left[1-\exp \left(-\lambda_{n} t\right)\right]\right\} \\
\dot{\varepsilon}_{\mathrm{vp}}=(1-\beta / 3) \Gamma\left(f / \sigma_{y}^{0}\right)^{N} \\
f=(1-\alpha / 3) \sigma-k\left(\varepsilon_{e}^{\mathrm{vp}}\right) \\
g=(1-\beta) \sigma
\end{array}\right.
$$

$$
k\left(\varepsilon_{\mathrm{e}}^{\mathrm{vp}}\right)=k_{0}+k_{1}\left(1-\exp \left(-k_{2} \varepsilon_{\mathrm{e}}^{\mathrm{vp}}\right)\right)
$$

It is obviously shown from Eq. (13) that viscoelastic strain can be obtained directly as soon as viscoelstic parameters are determined. The viscoplastic flow rule and internal state variables evolution consists of a first-order ordinary differential equations (ODEs) which cannot be treated 
analytically, in this study the backward difference formulas (BDF) is applied to solve the stiff ODEs, the procedure for the determination of viscoplastic strain is discussed below.

Write $\dot{\varepsilon}_{11}^{\text {vp }}$ to $\dot{y}$ implies:

$$
\dot{y}=\frac{y_{i+1}-y_{i}}{\Delta t}=(1-\beta / 3) \Gamma\left(\frac{(1-\alpha / 3) \sigma-k\left(y_{i+1}\right)}{\sigma_{y}^{0}}\right)^{N}
$$

Rewrite Eq. (15) implies:

$$
y_{i+1}-\Delta t(1-\beta / 3) \Gamma\left(\frac{(1-\alpha / 3) \sigma-k\left(y_{i+1}\right)}{\sigma_{y}^{0}}\right)^{N}-y_{i}=0
$$

Write $y_{i+1}$ to $z$ implies:

$$
f(z)=z-\Delta t(1-\beta / 3) \Gamma\left(\frac{(1-\alpha / 3) \sigma-k(z)}{\sigma_{y}^{0}}\right)^{N}-y_{i}=0
$$

Newton-Raphson scheme is used here in order to solve Eq. (17):

$$
\begin{aligned}
z_{\text {new }} & =z-f(z) / f^{\prime}(z) \\
& =z-\frac{z-\Delta t(1-\beta / 3) \Gamma\left(\frac{(1-\alpha / 3) \sigma-k(z)}{\sigma_{y}^{0}}\right)^{N}-y_{i}}{1+N \Delta t(1-\beta / 3) \Gamma\left(\frac{(1-\alpha / 3) \sigma-k(z)}{\sigma_{y}^{0}}\right)^{N-1} \frac{k_{1} k_{2} \exp \left(-k_{2} z\right)}{\sigma_{y}^{0}}}
\end{aligned}
$$

The values of $z$ getting from Eq. (18) is the uniaxial viscoplatic strain, the numerical computation wrote above can be achieved by MATLAB procedure.

\section{Parametric study}

The procedure for the determination of the viscoelastic-viscoplastic model parameters of modified double base propellant has been thoroughly discussed by WANG[14] based on creeprecovery tests. The obtained viscoelastic and viscoplastic model parameters at the reference temperature are listed in Table 1 and Table 2.

TABLE 1. Viscoelastic model parameters

\begin{tabular}{ccccc}
\hline$n$ & 0 & 1 & 2 & 3 \\
\hline$D_{n}\left(\mathrm{MPa}^{-1}\right)$ & 0.00369 & 0.00276 & 0.00212 & 0.00192 \\
$\lambda_{n}\left(\mathrm{~s}^{-1}\right)$ & & 0.1 & 0.01 & 0.001 \\
\hline
\end{tabular}

TABLE 2. Viscoplastic model parameters

\begin{tabular}{ccccccc}
\hline$\Gamma$ & $k_{0}(\mathrm{MPa})$ & $k_{1}(\mathrm{MPa})$ & $k_{2}$ & $N$ & $\alpha$ & $\beta$ \\
\hline 0.0010 & 0.0599 & 5.011 & 20.782 & 2.6544 & 0.30 & 0.15
\end{tabular}

ABU AL-RUB[15] presents the results of a parametric study for all of the viscoplastic material parameters, mainly analyses the effet on strain-stress response under constant strain rate. In this section, the parametric study focus on the creep response of modified double base propellant. 
In order to more clearly analyse the effect of various parameters on the viscoplastic creep strain, the viscoplastic parameters are adjusted based on the given parameters in Table 1 ang Table 2. As shown in Fig. 1, Fig. (a) shows that the greater values of viscoplastic potential energy parameter $\Gamma$ correspond to more flow, especially in the first creep stage; Fig. (b), Fig. (c) and Fig. (d) show that the greater values of the hardening function parameter $k_{0}, k_{1}$ and $k_{2}$ result in less flow, and are best understood by understanding the hardening function $k$, which is shown in Eq. (7); Fig. (e) shows that the greater values of viscoplastic potential energy parameter $N$ correspond to less flow.

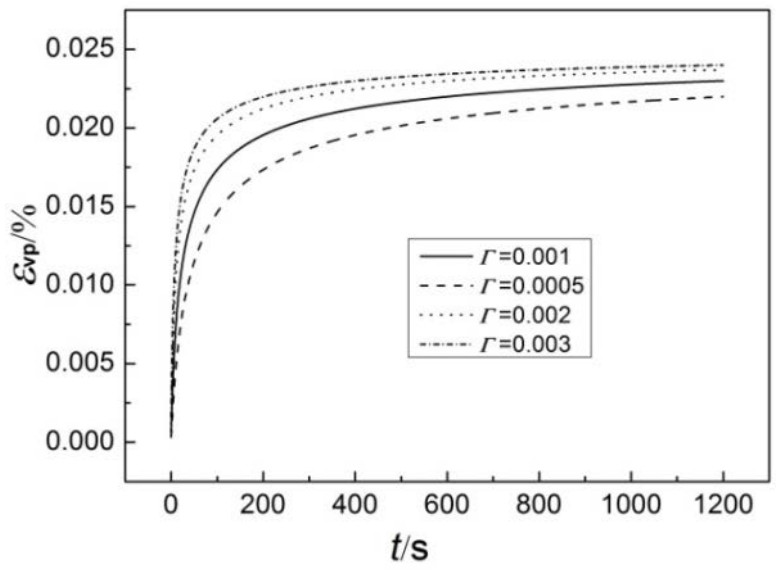

Fig. (a) Effect of parameter $\Gamma$

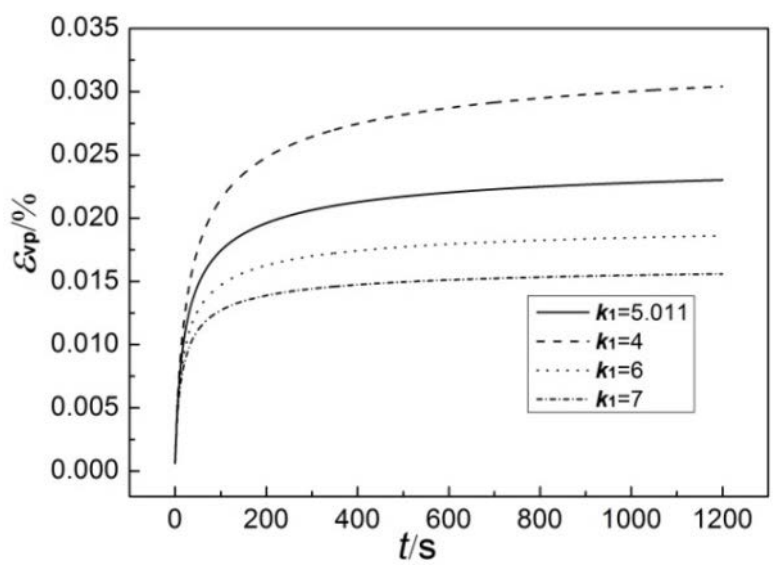

Fig. (c) Effect of parameter $k_{1}$

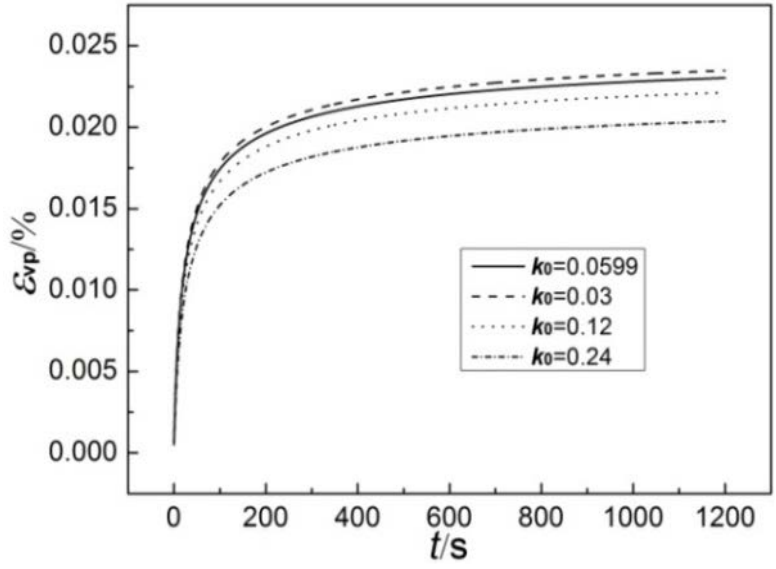

Fig. (b) Effect of parameter $k_{0}$

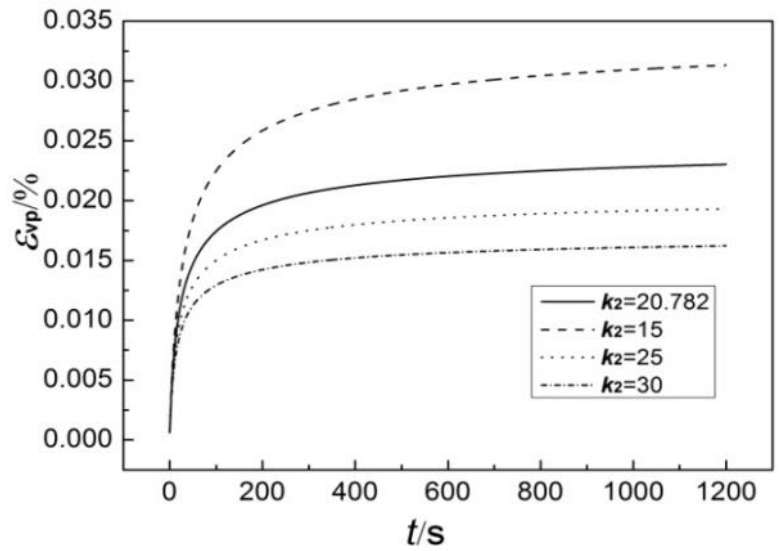

Fig. (d) Effect of

parameter $k_{2}$

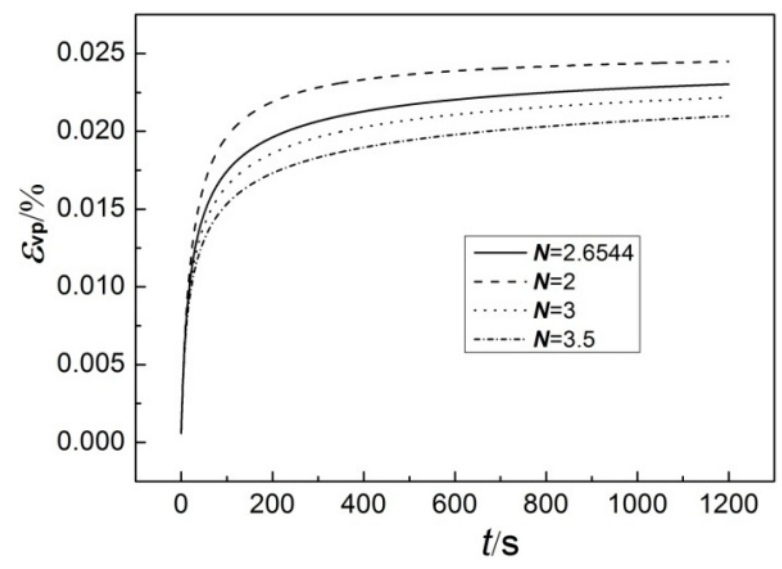

Fig. (e) Effect of parameter $N$

FIGURE 1. Effect of the viscoplastic parameters to viscoplastic creep strain 


\section{Conclusion}

1) One dimensional form of viscoelastic-viscoplastic constitutive model of modified double base propellant is derived for the convenience of numerical calculation, which is adapted to the uniaxial creep conditions.

2) The backward difference formulas (BDF) were applied to solve the stiff ODEs consisted by the viscoplastic flow rule and internal state variables evolution, the creep strain can be obtainded through MATLAB procedure.

3) Parametric study on the effect of viscoelastic material parameters show that the greater values of viscoplastic potential energy parameter $г$ correspond to more flow while $N$ correspond to more flow, the greater values of the hardening function parameter $k_{0}, k_{1}$ and $k_{2}$ result in less flow.

\section{References}

[1] XU J S, CHEN X, WANG H L, et al. Thermo-damage-viscoelastic constitutive model of HTPB composite propellant [J]. International Journal of Solid and Structures, 2014, 51:3209-3217.

[2] DENG K, YANG J H, HUANG W W, et al. A new method to obtain shear modulus of solid propellant[J]. Acta Astronautica, 2011, 69:440-444.

[3] SUN C X, XU J S, CHEN X, et al. Strain rate and temperature dependence of the compressive behavior of Composite Modified double-base propellant[J]. Mechanics of Materials, 2015, 89: 3546.

[4] MENG H L. Research on numerical simulation method of structure integrity analysis for modified double base propellant grain[D]. Nanjing: Nanjing University of Science and technology, 2011.

[5] M. K. DARABI, R. K. ABU AL-RUB, E. MASAD, et al. Thermodynamic-based model for coupling temperature-dependent viscoelastic, viscoplatic, and viscodamage of constitutive behavior of asphalt mixtures[J] International Journal for Numerical and Analytical Methods in Geomechanics, 2012, 36: 817-854.

[6] M. K. DARABI, R. K. ABU AL-RUB, E. MASAD, et al. A thermo-viscoelastic-viscoplasticviscodamage model for asphalt material[J] International Journal of Solid and Structures, 2011, 48: 191-207.

[7] J. S. KIM, A. H. MULOANA. A combined viscoelastic-viscoplastic behavior of particle reinforced composites[J]. International Journal of Solid and Structures 2010, 47:580-594.

[8] ZHU Y T, SUN L, ZHU H R, et al. A constitutive model of viscoelastic-viscoplatic solid based on thermodynamics theory [J]. Chinese Quarterly of Mechanics, 2010, 31(4):449-459.

[9] ZHU H R, SUN L, ZHU Y T. Viscoelastic-viscoplatic damage constitutive model based on thermodynamics for asphalt mixtures[J]. Chinese Journal of Highway and Transport, 2013, 26(3): 57-64.

[10] J LEMAITRE, J.-L. CHABOCHE. Mechanics of Solid Materials[D]. London: Cambridge University Press, 1990.

[11] S. H. DESSOUKY, Multiscale approach for modeling hot mix asphalt[D]. College station: Texas A\&M University, 2005.

[12] R. K. ABU AL-RUB, G. Z. VOYIADJIS. Gradient-enhanced coupled plasticity-anisotropic damage model for concrete fracture: computational aspects and applications[J]. International Journal of Damage Mechanics, 2009, 18 (2), 115-154. 
[13] M. K. DARABI, R. K. ABU AL-RUB, E. MASAD, et al. A modified viscoplastic model to predict the permanent deformation of asphaltic materials under cyclic-compression loading at high temperatures[J]. International Journal of Plasticity, 2012, 35:100-134.

[14] WANG H L, XU J S, CHEN X, et al. Research on the viscoelastic-viscoplastic constitutive model of modified double base propellant [J].Acta Aeronautica et Astronautica Sinica,2016,37(x):xxx-xxx.

[15] R. K. ABU AL-RUB, E. MASAD, C. W. HUANG. Improving the sustainability of asphalt pavements through developing a predictive model with fundamental Material Properties [R]. Springfield, Virginia: National Technical Information Service, 2005. 